\title{
VARIA:
}

\section{Johann Amos Comenius und Deutschland. Grundzüge einer Rezeptionsgeschichte bis 1945}

\author{
Andreas LISCHEWSKI ${ }^{\mathrm{a}}$
}

aAlanus Hochschule, Comenius-Forschungsstelle und Comenius-Forschungsbibliothek, Alfter bei Bonn, Deutschland

Comenius hat immer wieder betont, dass er von deutschen Pädagogen viele Anregungen übernommen habe. Aber: Er hat Deutschland auch viel gegeben.

In den letzten knapp 400 Jahren hat er immer wieder pädagogische Wissenschaftler und Praktiker dazu inspiriert, nicht nur über eine wirksamere Unterrichtsmethodik nachzudenken, sondern auch über die Frage, was eine durchdachtere Schulbildung zu einer Verbesserung der menschlichen Angelegenheiten insgesamt beizutragen vermag. Denn dass der Schule sowohl für eine angemessene Ausbildung der Kinder und Jugendlichen als auch für die Herbeiführung besserer gesellschaftlicher Verhältnisse eine zentrale Rolle zukommt, ist seit Comenius eine Überzeugung, die eigentlich alle modernen Pädagogen bis heute teilen:

„Es konzentriert sich also die ganze Hoffnung auf ein besseres Zeitalter auf die eine und einzige rechte Erziehung der Jugend (ad solam unam Juventutis rectam Educationem) und damit auch auf die ordnungsgemäß eingerichteten Schulen (scholas recte constitutas). [...] Wir müssen es nämlich so machen, dass vor der Erneuerung der Kirchen und des Staates zuerst die Schulen als die Werkstätten des Lichtes reformiert werden. [...] Denn dann werden wir sehen, wie sich die Strahlen dieses Lichtes der Weisheit aus den Schulen schon bald machtvoll auch über die Kirche, die weltliche Ordnung und überhaupt über den ganzen Umkreis der Dinge ausbreiten werden, um alles größer und besser, schöner und liebenswerter zu machen.“ (Panorthosia XXII, 6.9 [323/584 f.])

Gemäß der thematischen Vorgabe, die Geschichte der Comeniusrezeption in Deutschland in ihren Grundzügen darzustellen, werde ich meinen Überblick historisch gliedern, also abschnittsweise vom 17. Jahrhundert bis in die jüngste Gegenwart fortschreiten. Dabei werde ich allerdings weniger die Vollständigkeit der Darstellung anstreben, als vielmehr die zentralen Entwicklungen und Einsichten zu fokussieren versuchen.

\section{Rezeptionslinien bis zum Beginn des 20. Jahrhunderts}

Die deutsche Comeniusrezeption zwischen dem 17. und dem frühen 20. Jahrhundert wird häufig negativ bewertet und solcherart als eine Verfallsgeschichte beschrieben, die das geniale Erbe des großen Tschechen im Laufe der Zeit unzulässigerweise auf schulpädagogische und 
didaktische Errungenschaften verkürzt habe. Seit Klaus Schaller das entsprechende Wort von der ,verkehrenden Inanspruchnahme“ (1962, S. 373 ff.) des Comenius durch die Didaktiker ins Spiel gebracht hatte, wurde dieser Topos umfangreich rezipiert, um die entstellenden Interpretationen der Vergangenheit von der je eigenen Darstellung säuberlich zu scheiden, die dann endlich eine sauthentische> Auslegung des Comenius bieten sollte (Lischewski, 2013, S. 386 f.).

Viele dieser, zu einem gewissen Dualismus und Dogmatismus neigenden Urteile beruhen auf einer geringschätzenden Unkenntnis der tatsächlichen Leistungen, die von den Comeniusinterpreten der Vergangenheit in oft mühsamer Weise erarbeitet wurden - bei allen zugestandenen Unzulänglichkeiten, die wir heute rückblickend durchaus wohlwollend belächeln dürfen. Dennoch ist die Zeit gekommen, um hier ein ausgewogeneres Urteil zu fällen.

1.1 Blicken wir in das 17. und 18. Jahrhundert, so hat sich die deutsche Comenius-Rezeption sicherlich zunächst nicht unwesentlich auf dessen Schulbücher bezogen, besonders auf die 1631 veröffentlichte Janua linguarum sowie auf den Orbis sensualium pictus von 1658 . Von beiden Werken erschienen in Deutschland zahlreiche Ausgaben und Umarbeitungen, die bis weit in das 19. Jahrhundert hinein in Gebrauch waren. Sie begründeten des Comenius Weltruf als genialer Schulbuchautor, wobei insbesondere die pansophischen Zusammenhänge für spätere Zeiten nicht mehr ohne weiteres verständlich waren. Es ist diese Tatsache jedoch schon so häufig beschrieben und wiederholt worden (Kvačala, 1904; Michel, 1973), dass sie hier nicht nochmals in epischer Breite dargestellt werden muss.

Umso wichtiger ist der Hinweis, dass die deutsche Comenius-Rezeption schon damals durchaus breiter aufgestellt war. So ist schon früh auf die enge Geistesverwandtschaft zwischen Comenius und dem deutschen Pietismus hingewiesen worden. Bereits Philipp Jakob Spener (1635-1705) hatte die comenianischen Schriften nämlich - bei aller Kritik im Einzelnen - sehr geschätzt. Ferner wäre hier August Hermann Francke (1663-1724) zu erwähnen, da nicht zuletzt auf sein Interesse an den Slaven die „Comeniusrenaissance“ (Winter, 1955, S. 277) in Halle zurückgeführt werden kann. Denn in ihrem Gefolge wurden zahlreiche, insbesondere theologische und erbauliche Schriften des Comenius nachgedruckt und überliefert. Auch lagerte in der Bibliothek des Waisenhauses das Manuskript der Consultatio Catholica, von welchem Johann Franz Buddeus (1667-1729) bekanntlich die ersten zwei Teile veröffentlichte - auch wenn eine geplante Gesamtausgabe der comenianischen Werke damals nicht zustande kam. Dass Comenius später in den religiösen Traditionen der Herrnhuter Brüdergemeine fortwirkte und dort - etwa über Paul Eugen Layritz (1707-1788) - das Schul- und Ausbildungswesen entscheidend mitprägte (Doerfel, 1992), sei genauso am Rande vermerkt wie die Tatsache, dass es sogar einen Einfluss seiner Physik auf die sich damals in Halle neu konstituierende Medizin gegeben zu haben scheint (Geyer-Kordesch, 2000, S. 89).

Umgekehrt scheinen mir zwei andere Denker, die man gemeiniglich als vorbildhafte Ausnahmeinterpreten des 18. Jahrhunderts lobt, in ihrer Bedeutung weitgehend überschätzt zu werden. Dass der noch junge Gottfried Wilhelm Leibniz (1646-1716) in voller 
Begeisterung ein Lobgedicht auf Comenius verfasst hat, das auch bereits eine deutliche Kenntnis seiner pansophischen und physikalischen Anschauungen widerspiegelt, ist genauso bekannt wie die Tatsache, dass es zahlreiche Ähnlichkeiten im philosophischen Denken dieser beiden Barocksystematiker gibt. Dennoch ist schon früh darauf hingewiesen worden, dass das Denken von Leibniz später in mancherlei Hinsicht eine andere Richtung genommen habe (Kvačala, 1904, S. 146) und solcherart der von den Comeniologen so gerne konstruierte Einfluss des Comenius auf Leibniz viele bleibende Unterschiede deutlich verkenne (Bittner, 1930, S. 83). Und ähnlich zurückhaltend ist wohl auch die Rolle Johann Gottfried Herders (1744-1803) zu beurteilen. Er dürfte insgesamt nur sehr wenige Texte des Comenius gekannt haben; und selbst seine Darstellung der Panegersia im 57. Humanitätsbrief scheint sich auf wenige ausgewählte Kapitel zu beschränken, die dann auch noch im Wesentlichen nur nach den Randkolumnen zitiert werden (Drews, 1990, S. 59 f.). Auch darf nicht verkannt werden, dass Herder wirkungsgeschichtlich - und zwar nicht gerade zum Vorteil einer unbefangenen Comeniusdeutung - durch sein „Stereotyp“ (Wirrer, 1996) von den kriegerischen Deutschen und den friedliebenden Slaven bedeutsam wurde. An der Auffassung, dass die ComeniusInterpretationen von Leibniz und Herder historisch angemessener seien als diejenigen seiner Kritiker, wird man also einigen Zweifel hegen dürfen.

$\mathrm{Zu}$ eben diesen Kritikern, die von der traditionellen Comeniologie wegen ihrer teilweise recht überzogenen Kritik oft sehr pauschal abgeurteilt werden, gehörte aber auch Johann Christoph Adelung (1832-1806), dessen Comeniusdarstellung in der Tat nicht gerade wohlwollend ausfiel. Dennoch werden wir heute nicht alle Kritikpunkte als völlig unberechtigt zurückweisen dürfen. Bedenkt man etwa des Comenius starre Fixierung auf die schlechthin eine Wahrheit (Lischewski, 2019), dann erscheint die Warnung Adelungs, dass Comenius seine Weltsicht als für alle Menschen verbindlich vorschreiben wollte (Adelung, 1785, S. 196-198 u. ö.), als durchaus genauso plausibel wie der Vorwurf, dass das Beleidigende in den Beschimpfungen gegen das Haus Österreich und den Papst nicht recht zu seiner vielgepriesenen Friedfertigkeit zu passen scheint (ebd., S. 216 f.).

Es braucht keiner weiteren Ausführungen, um einsichtig zu machen, dass die verschiedenen Comeniusinterpretationen schon des 17. und 18. Jahrhunderts weit weniger pauschal be- und verurteilt werden dürfen, wie es verschiedentlich gerne getan wird. Eine nüchternere Aufarbeitung dieser Rezeptionslinien scheint darum dringend angeraten.

1.2 Wegweisende Entwicklungen, die bisher nur selten angemessen gewürdigt wurden, lassen sich aber auch für das >lange 1 19. Jahrhundert nachweisen. Das betrifft vor allen Dingen die Wiederentdeckung der Opera Didactica Omnia, die nunmehr in ihrer Gesamtheit immer breiter rezipiert wurden. Denn obschon das Bedürfnis der Lehrerschaft nach Vorschlägen zur praktischen Unterrichtsgestaltung in dieser Zeit noch durchweg leitend blieb, begann man sich jetzt zunehmend auch mit den pansophischen Programmschriften und den umfassenderen Reformzielen des Comenius auseinanderzusetzen.

Für die faktische Kenntnis comenianischer Gedanken kaum zu unterschätzen sind zunächst die zahlreichen Publikationen, die jetzt im deutschen Sprachgebiet in stetig wachsender Zahl verbreitet wurden. Nach einer ersten sehr wohlwollenden Darstellung bei Friedrich Heinrich 
Christian Schwarz (1766-1837), die in mancherlei Hinsicht bereits das protestantisch dominierte Deutungsschema der nachmaligen Zeit charakteristisch vorwegnahm - hier der fromme Enthusiasmus des vorbildhaften Comenius, dort seine verdammenswerten Neider (Schwarz, 1813, S. 330) -, war es dabei vor allen Dingen der stark vom Pietismus beeinflusste Karl von Raumer (1783-1865), der dieses Comeniusbild nachhaltig an die späteren Generationen vermittelte. Obwohl er ausdrücklich betont, dass unter Vernachlässigung biographischer Einzelheiten ,vorzüglich die pädagogische Wirksamkeit“ (von Raumer, 1843, S. 50 f.) des Comenius beschrieben werden sollte, setzt nämlich auch von Raumer zuletzt auf die Darstellung jener ,großen ehrwürdigen Leidensgestalt“, die - heimatlos umherirrend dennoch unermüdlich und mit ,ausdauernder glaubensstarker Treue“ am Projekt einer „gottgefälligeren Generation“ gearbeitet habe (ebd., S. 91). Andererseits aber, und auch das ist wirkungsgeschichtlich bedeutsam, widmete er nunmehr einen Großteil seiner Ausführungen dem Corpus der Opera Didactica Omnia, sie dabei „als reichste Schatzkammer scharfsinniger und tiefer pädagogischer Gedanken“ (ebd., S. 52-90) vorstellend. Persönlichkeitsbezogene Stilisierung und Ausweitung der sachlichen Kenntnisse gingen hier erstmalig ein intimes Bündnis ein - und wurden in dieser Doppelung maßgeblich für die Comeniusrezeption der Folgezeit. Insbesondere nach der Einführung der „Geschichte der Pädagogik" als eines obligatorischen Fachs der Lehrerbildung - 1863 in Gotha, 1866 in Bayern und 1872 in Preußen (Bühler, 2012, S. 687 f.) - entstanden zahlreiche Lexikon- und Handbuchartikel, die sich fast durchgehend auf das Werk von Raumers zurückbezogen und in diesem Sinne zumeist auch seine Wertung übernahmen. Bis etwa 1879 war dann die Kanonbildung pädagogischer Klassiker weitgehend abgeschlossen: und Comenius gehört seitdem zu den mit am häufigsten zitierten Autoren - bis in die Gegenwart (Hild, 2018, S. 100 f., 140; Winkler, 1994, S. 153). Allein um dieser Entwicklung willen gilt es, die Leistungen des 19. Jahrhunderts angemessen zu würdigen; und doch erschöpft sie sich nicht darin.

Da wäre beispielsweise auf die verschiedenen Textausgaben zu verweisen, mittels derer das comenianische Gedankengut nun ebenfalls zunehmend bekannt gemacht wurde. Ausgehend von der sich allmählich durchsetzenden Überzeugung, dass die theoretischen Schriften des Comenius weit wichtiger seien als seine Schulbücher (Schiller, 1887, S. 165; Nebe, 1903, S. 918) erscheinen solcherart vor allen Dingen zahlreiche Übersetzungen der Didactica Magna, darüber hinaus aber auch weitere Schriften aus den Opera Didactica Omnia sowie verschiedene nicht-pädagogische Texte - wofür hier stellvertretend die berühmten Ausgaben der Naturkunde durch Joseph Reber (1898) und des Allgemeinen Weckrufs durch Herbert Schönebaum (1924) genannt werden mögen.

Ferner erscheinen jetzt mehrere Monographien - häufig hervorgegangen aus Dissertationen -, mittels derer das comenianische Werk nunmehr auch systematisch-interpretatorisch tiefer erschlossen wurde. Da wird etwa ausführlich seine Theologie erläutert (Criegern, 1881), die systematische Grundlegung der Pädagogik analysiert (Müller, 1887) oder auch das naturphilosophische Denken dargestellt (Kvacsala, 1886). Von besonderem Interesse sind in diesem Zusammenhang aber auch die Arbeiten über die Pansophie des Comenius, die jetzt 
ebenfalls verstärkt bearbeitet wird - allen voran die Arbeit von Gustav Beißwänger, deren Bedeutung sich keinesfalls darin erschöpft, erstmalig alle von Comenius damals bekannten Schriften zur Pansophie in ihrer chronologischen Entwicklung umfassend dargestellt zu haben. Interessant ist vielmehr, dass bereits hier auch schon deutlich auf einige Schwachstellen des pansophischen Denkens hingewiesen wird, die man später leichtfertig vergessen hat. So hat Beißwänger bereits ganz klar gesehen, dass Comenius die Heiden selbstverständlich niemals in letzter Konsequenz als ebenbürtige Gesprächspartner ansehen konnte (Beißwänger, 1904, S. 59). Auch kritisierte er deutlich den von Comenius wiederholt angemeldeten Wahrheitsanspruch einer absoluten Unfehlbarkeit der Pansophie in ihrer vollendeten Form, die seiner Überzeugung nach zu noch mehr Widerspruch reizen müsse als die „Dogmen der mittelalterlichen Kirche“ (ebd., S. 70), weil Comenius die Bezogenheit seiner pansophischen Aussagen auf Zeit systematisch nicht denken könne.

Auch die Erforschung der comenianischen Biographie machte $\mathrm{zu}$ dieser Zeit enorme Fortschritte. So wurden etwa die zahlreichen biographischen Hinweise, die Comenius in seine Opera Didactica Omnia eingestreut hatte, nunmehr systematisch erfasst und ausgewertet (Palacký, 1929). Hernach ermöglichte der Fund eines ausführlichen Briefwechsels sowie weiterer Schriftstücke aus dem Umkreis der Brüdergemeine in Polen, insbesondere die Ereignisse aus der zweiten Lebenshälfte des Comenius genauer zu rekonstruieren (Gindely, 1855). Den nachhaltigsten Einfluss auf die Comeniusforschung übte jedoch der slowakische Kirchen- und Kulturhistoriker Ján Kvačala aus, der in zahlreichen Schriften und Abhandlungen das vielfältig vorhandene Material $\mathrm{zu}$ Comenius arkribisch sichtete, um es zu einem in sich stimmigen Gesamtbild seines Lebens und seiner Werke zusammenzufügen. Seine umfassend recherchierte Biographie (Kvacsala, 1892) gilt bis heute als Meilenstein der Comeniusforschung.

Nimmt man noch die zwei bedeutendsten Institutionen dieser Zeit hinzu, die sich ebenfalls zentral um die Verbreitung comenianischer Gedanken und Schriften bemüht hatten - die 1871 erfolgte Stiftung der Comenius-Bibliothek durch den Leipziger Lehrerverein (Ritzi, 1994) einerseits sowie die 1891 gegründete Berliner Comenius-Gesellschaft (Korthaase, 1993) andererseits - dann ist mehr als offensichtlich, welche Verdienste das >lange< 19. Jahrhundert für die Comeniusforschung gehabt hat. Und dass bei aller Bewunderung für diesen Mann, den man „fraglos als einen der größten Pädagogen aller Zeiten“ zu würdigen wusste, zugleich kritisch und realistisch vor einer bedenklichen „Gefahr der Überschätzung“ seines Werkes gewarnt werden konnte (Ziegler, 1895, S. 164; Beißwänger, 1904, S. 97), steigert die Bedeutung dieser Zeit nochmals. Gerade in dieser Hinsicht scheint sie nämlich unserer Zeit durchaus weit voraus gewesen zu sein!

1.3 Obwohl nach der Jahrhundertwende und insbesondere nach dem I. Weltkrieg das Interesse an Comenius deutlich spürbar zurückging, erschienen noch bis zum Ende der Weimarer Republik immer wieder auch rezeptionsgeschichtlich interessante Texte.

Besonders aufschlussreich ist hier zunächst jene kulturkritische Wendung der Interpretation, in deren Wortwahl man schon die spätere Comeniusdeutung der 1960er Jahre zu vernehmen meint. So sprach Erwin Hanslik unter dem Eindruck der Kriegsereignisse von einem 
„,vollständigen Bankrott der Einzelwissenschaften“ und ihres „technischen Spezialwissens“, das durch eine „Wissenschaft vom Ganzen der Welt“ überwunden werden müsse, falls man wieder $\mathrm{zu}$ einer friedlichen Einigung der Menschen zurückfinden wolle (Hanslik, 1920, S. 127, 135 u.ö.). Und nicht minder finden wir schon zu dieser Zeit den unzweideutigen Hinweis, dass die comenianische Bildungstheorie auf eine „umfassende Welterkenntnis“ ziele, durch welche „das Individuum aus sich selbst hinausführt“" werden solle (Staedke, 1930, S. $107 \mathrm{f}$.$) .$

Dass die pansophische Gesamtkonzeption einer ganzheitlichen Weltsicht mit vielen modernen, häufig einseitig subjektorientierten Bildungstheorien nur schwerlich in Übereinstimmung zu bringen ist, war also auch hier schon durchaus bekannt; und es war bekannt - um das mit aller Deutlichkeit zu sagen -, ohne dass man die Consultatio Catholica und die Pampaedia insbesondere gekannt hätte.

1.4 Überblickt man die Entwicklung zwischen dem 17. und dem frühen 20. Jahrhundert so wird man sagen können, dass diese für die Kenntnis der comenianischen Werke und für die Verbreitung seiner Ideen viele wertvolle Beiträge geleistet hat. Freilich war die Rezeption dabei zunächst überwiegend pädagogisch ausgerichtet. Doch wird man selbst diesem Rezeptionsstrang eine wichtige Bedeutung beizumessen haben, da er über die Wiederentdeckung der Opera Didactica Omnia in ihrer Gesamtheit auch der Rezeption der comenianischen Pansophia vorgearbeitet hat - ganz abgesehen von der Tatsache, dass die Lehrer immer schon zu den wesentlichen Multiplikatoren gehörten, die das Andenken an Comenius überhaupt aufrechterhalten haben.

Man wird daher nicht sagen können, dass Comenius in dieser gut 250 Jahre währenden Entwicklung einfach nur schulpädagogisch `verkürzt` worden sei. Im 18. Jahrhundert spielte das theologisch-erbauliche Erbe des Comenius eine nicht unbedeutende Rolle; und im 19. Jahrhundert kam die im engeren Sinne wissenschaftliche Beschäftigung mit Comenius - seiner Biographie und seinem Werk - hinzu. Der damals nachhaltig einsetzende Aufschwung der Auseinandersetzung mit Comenius schuf damit bedeutsame Vorarbeiten, auf denen auch die Nachkriegscomeniologie der 1960er Jahre später nicht unerheblich fußen wird - auch wenn sie sich dieses nicht immer ausdrücklich eingestand.

\section{Intermezzo: Comenius zwischen 1933 und 1945}

2.1 Die Comeniusrezeption während der Zeit der nationalsozialistischen Herrschaft liegt noch weitgehend im Dunklen. Doch spricht vieles dafür, dass man hier sehr umsichtig und vor allen Dingen sehr differenziert wird hinschauen müssen. Die Vorstellung etwa, dass Comenius vom nationalsozialistischen Regime als Slave bekämpft und in propagandistischer Verzerrung als Deutscher vereinnahmt worden sei (Korthaase, 2009, S. 200 f., 210 f.), lässt sich in dieser Pauschalität nicht aufrechterhalten. So wurde Comenius im Jubiläumsjahr 1942 überwiegend als >Mähre< bezeichnet, ohne dass diese Tatsache negativ kommentiert worden wäre; und zugleich darf nicht vergessen werden, dass die Vereinnahmung als `Deutscher`, wo sie denn auftauchte, ausgerechnet auf den vielzitierten Herder zurückgriff, der ihn erstmals als einen Mann der deutschen Nation bezeichnet hatte. 
Damit ist freilich nicht ausgeschlossen, dass der Zeitgeist auch in den Comeniusdeutungen sichtbare Spuren hinterließ. So finden wir in dieser Zeit etwa einen Aufsatz über den comenianischen Begriff der Volkes, der allerdings relativ problemlos an Vorarbeiten aus der Weimarer Republik anknüpfte (Schönebaum 1937). Auch eine Münchner Dissertation, die Comenius in die Geschichte der Mütterschulung einordnet, kann ihren Zeitbezug kaum leugnen, ist aber aufgrund des Interesses, das die vergangenen Jahrzehnte am Informatorium der Mutterschul gehegt hatten, als solche ebenfalls nicht notwendig ungewöhnlich (Rahner, 1936, S. 7-10). Aufgearbeitet sind diese Zusammenhänge bis heute nicht. Mit pauschalisierenden Urteilen sollte man sich darum aber auch hier vorerst zurückhalten.

2.2 Es fiel in diese Zeit aber zumindest ein Ereignis, das für die Comeniusforschung insgesamt von herausragender Bedeutung werden sollte: nämlich die Auffindung des - längst verloren geglaubten - pansophischen Hauptwerkes des Comenius, der Consultatio Catholica de rerum humanarum emendatione, im Archiv der Hauptbibliothek des Franckeschen Waisenhauses in Halle durch Dmitrij Tschižewskij in den 1930er Jahren (Tschižewskij, 1972).

Dieser Fund hat in der Folgezeit viel Aufsehen erregt, und er war von viel zu internationaler Bedeutung, als dass er hier für die Geschichte der spezifisch deutsch-sprachigen Comeniusrezeption in Anspruch genommen werden dürfte. Dennoch ist der Fundort ein erneutes Indiz dafür, dass es bereits im 18. Jahrhundert über die im engeren Sinne didaktischen Rezeption hinaus auch ein theologisch-pietistisches Interesse an Comenius gegeben hatte. Zugleich wirft er aber auch die nicht minder interessante Frage auf, warum das Manuskript hier zuletzt vergessen wurde und woran das ursprüngliche Interesse an Comenius dann zuletzt dennoch gescheitert ist. Auch hier liegt noch vieles im Dunkeln. (Fortsetzung folgt)

\section{Literatur}

ADELUNG, Christoph Johann, 1785. Geschichte der menschlichen Narrheit, oder Lebensbeschreibungen berühmter Schwarzkünstler, Goldmacher, Teufelsbanner, Zeichenund Liniendeuter, Schwärmer, Wahrsager, und anderer philosophischer Unholden. Leipzig: Weygand.

BEISSWÄNGER, Gustav, 1904. Amos Comenius als Pansoph. Eine historischphilosophische Untersuchung. Stuttgart: Kohlhammer.

BITTNER, Konrad, 1929, 1930. J. A. Comenius und G. W. Leibniz. Zeitschrift für slavische Philologie. 6 (1929), S. 115-145; 7 (1930), S. 53-93.

BÜHLER, Patrick, 2012. „Vom Nutzen und Vorteil der Historien für das Leben.“ Vorreden und Einleitungen zu deutschen Geschichten der Pädagogik des 19. Jahrhunderts. Paedagogica Historica. 48 (2012), S. 676-691.

COMENIUS, Johann Amos, 1966. De emendatione rerum humanarum Consultationis Catholicae Pars Sexta, Panorthosia. De rerum humanarum emendatione Consultatio Catholica (Editio princeps). Tom. II, hg. v. Otokar Chlup, Prag 1966, S. 205/Sp. $356-$ 


\section{S. 378/Sp. 695 .}

CRIEGERN, Hermann Ferdinand von, 1881. Johann Amos Comenius als Theolog. Ein Beitrag zur Comeniusliteratur. Leipzig \& Heidelberg: C.F. Winter.

DOERFEL, Marianne, 1992. Zur Übernahme der Pädagogik des Comenius durch Paul Eugen Layritz. Unitas Fratrum. 32 (1992), S. 65-90.

DREWS, Peter, 1990. Herder und die Slaven. Materialien zur Wirkungsgeschichte bis zur Mitte des 19. Jahrhunderts. München: Otto Sagner.

GEYER-KORDESCH, Johanna, 2000. Pietismus, Medizin und Aufklärung in Preußen im 18. Jahrhundert. Das Leben und Werk Georg Ernst Stahls (= Hallesche Beiträge zur europäischen Aufklärung 13). Tübingen: Niemeyer. ISBN 978-3484810136.

GINDELY, Anton, 1855. Über des Johann Amos Comenius Leben und Wirksamkeit in der Fremde. Sitzungsberichte der der philosophischhistorischen Classe der kaiserlichen Akademie der Wissenschaften XV (Separatdruck). Wien: Hof- und Staatsdruckerei.

HANSLIK, Erwin, 1920. Pansophia und Kulturforschung. In: PAQUET, Alfons, Hg. Der Rhein als Schicksal oder: Das Problem der Völker. Bonn: Cohen, S. 117-144.

HILD, Anne, 2018. „Helden und Denker“ der Pädagogik im Spiegel ihrer Fachlexika von 1774 bis 1945 (= Erziehungswissenschaftliche Studien 2). Göttingen: Universitätsverlag. ISBN 978-3-86395-357-7.

KORTHAASE, Werner, 1933. Korthaase: Die Berliner internationale Comenius-Gesellschaft zur Pflege der Wissenschaft und Volkserziehung (1891-1934). Berlin: Comenius-Zentrum der Humboldt-Universität.

KORTHAASE, Werner, 2009. Habent sua fata libelli. Das Schicksal des Hauptwerkes von J. A. Comenius. Studia Comeniana Sedlcensia. Bd. II, hg. v. Barbara Sitarska u. a. Siedlce: Wyd. Akademii Podlaskiej, S. 199-220. ISBN 978-83-7051-540-9.

KVACSALA, Johann, 1886. Über J. A. Comenius' Philosophie insbesondere Physik. Leipzig: Teubner.

KVACSALA, Johann, 1892. Johann Amos Comenius. Sein Leben und seine Schriften. Leipzig / Wien: Klinkhardt.

KVAČALA, Johannes, 1904. Die pädagogische Reform des Comenius in Deutschland bis zum Ausgange des XVII. Jahrhunderts Bd. 2 (= Monumenta Germaniae Paedagogica XXXII). Berlin: Hofmann \& Comp.

LISCHEWSKI, Andreas, 2013. Die Entdeckung der pädagogischen Mentalität bei Comenius. Zum Problem der anthropologischen Ermächtigung in der Consultatio Catholica. Paderborn: Schöningh. ISBN 978-3506777874.

LISCHEWSKI, Andreas, 2019. „Die Schrift stellt dem Frieden die Wahrheit voran.“ Chancen und Grenzen comenianischer Irenik. Comenius-Jahrbuch. 27 (2019), S. 13-64. ISBN 978-389665-859-3.

MICHEL, Gerhard, 1973. Schulbuch und Curriculum. Comenius im 18. Jahrhundert. Ratingen: Henn.

MÜLLER, Walter, 1887. Comenius. Ein Systematiker in der Pädagogik. Eine philosophischhistorische Untersuchung. Dresden: Bleyl \& Kaemmerer. 
NEBE, August, 1903. Art. >Comeniusく. Encyklopädisches Handbuch der Pädagogik. Hg. v. Wilhelm Rein. Langensalza: Beyer, S. 909-922.

PALACKÝ, F[rantišek], 1829. Ueber J. A. Comenius und seine Werke. Monatsschrift der Gesellschaft des vaterländischen Museums 3. Teil 1: S. 255-268, Teil 2: S. 330-343.

RAHNER, Elisabeth, 1936. Der Gedanke der Mütterschulung in seiner Entwicklung von Comenius bis zur Gegenwart. (Diss. München), Freiburg im Breisgau: Fürderer.

RAUMER, Karl von, 1843. Geschichte der Pädagogik vom Wiederaufblühen klassischer Studien bis auf unsere Zeit, Zweiter Theil. Stuttgart: Samuel Gottlieb Liesching.

RITZI, Christian, 1994. Comenianische Ideen verwirklichen. Der Beitrag der Leipziger Comenius-Bücherei zur Professionalisierung der Volksschullehrer. Comenius-Jahrbuch. 2 (1994), S. 37-52.

SCHALLER, Klaus, 1962. Die Pädagogik des Johann Amos Comenius und die Anfänge des pädagogischen Realismus im 17. Jahrhundert. Heidelberg: Quelle \& Meyer.

SCHILLER, Herman, 1887. Lehrbuch der Geschichte der Pädagogik. Leipzig: Reisland.

SCHÖNEBAUM, Herbert, 1937. Der Versuch einer Volkslehre bei Johann Amos Comenius. Ungarische Jahrbücher. 17 (1937), S. 120-128.

SCHWARZ, Friedrich Heinrich Christian, 1813. Erziehungslehre (Vierten Bandes Zweyte Abtheilung). Leipzig: Göschen.

STAEDKE, Hildegard, 1930. Die Entwicklung des enzyklopädischen Bildungsgedankens und die Pansophie des J. A. Comenius. Leipzig: Klinkhardt.

TSCHIŽEWSKIJ, Dmitrij, 1972. Wie ich die Handschriften der Pansophie fand. In: Ders.: Bohemica (= Kleinere Schriften II). München: Fink, S. 215-222.

WINKLER, Michael, 1994. Ein geradezu klassischer Fall. Zur Traditionsstiftung in der Pädagogik durch Klassiker. In: HORN, Klaus-Peter und WIGGER, Lothar, Hg. Systematiken und Klassifikationen in der Erziehungswissenschaf. Weinheim: Deutscher Studien Verlag. ISBN 978-3892714682.

WINTER, Eduard, 1955. Die tschechische und slowakische Emigration in Deutschland im 17. und 18. Jahrhundert. Beiträge zur Geschichte der hussitischen Tradition. Berlin: AkademieVerlag.

WIRRER, Jan, 1996. Stereotypien über europäische Völker in Herders >Ideen zur Philosophie der Geschichte der Menschheit . Germanoslavica. 3 (1996), S. 107-131.

ZIEGLER, Theobald, 1895. Geschichte der Pädagogik (= Handbuch der Erziehungs- und Unterrichtslehre für höhere Schulen I.1). München: Beck. 